\title{
Constraints on general neutrino interactions with exotic fermion from neutrino-electron scattering experiments
}

\author{
Zikang Chen, ${ }^{a}$ Tong $\mathrm{Li}^{b}$ and Jiajun Liao ${ }^{a}$ \\ ${ }^{a}$ School of Physics, Sun Yat-Sen University, \\ Guangzhou 510275, China \\ ${ }^{b}$ School of Physics, Nankai University, \\ Tianjin 300071, China \\ E-mail: chenzk7@mail2.sysu.edu.cn, litong@nankai.edu.cn, \\ liaojiajun@mail.sysu.edu.cn
}

ABSTRACT: The couplings between the neutrinos and exotic fermion can be probed in both neutrino scattering experiments and dark matter direct detection experiments. We present a detailed analysis of the general neutrino interactions with an exotic fermion and electrons at neutrino-electron scattering experiments. We obtain the constraints on the coupling coefficients of the scalar, pseudoscalar, vector, axialvector, tensor and electromagnetic dipole interactions from the CHARM-II, TEXONO and Borexino experiments. For the flavoruniversal interactions, we find that the Borexino experiment sets the strongest bounds in the low mass region for the electromagnetic dipole interactions, and the CHARM-II experiment dominates the bounds for other scenarios. If the interactions are flavor dependent, the bounds from the CHARM-II or TEXONO experiment can be avoided, and there are correlations between the flavored coupling coefficients for the Borexino experiment. We also discuss the detection of sub-MeV DM absorbed by bound electron targets and illustrate that the vector coefficients preferred by XENON1T data are allowed by the neutrino-electron scattering experiments.

Keywords: Beyond Standard Model, Neutrino Physics

ARXIV EPRINT: 2102.09784 


\section{Contents}

1 Introduction 1

2 The general neutrino interactions with exotic fermion via neutrinoelectron scattering

3 Neutrino-electron scattering experiments 4

3.1 CHARM-II 5

3.2 TEXONO 5

$\begin{array}{lll}3.3 & \text { Borexino } 6\end{array}$

4 Constraints from the experimental data $\quad 7$

4.1 Flavor-universal bounds $\quad 7$

4.2 Flavor-dependent bounds 8

5 Prospects at DM experiments $\quad 8$

6 Conclusion $\quad 12$

A Calculation of the differential cross section $\quad 12$

\section{Introduction}

The phenomenon of neutrino oscillations has been well confirmed by various neutrino experiments in the last two decades [1]. Since the explanation of neutrino oscillations requires nonvanishing neutrino masses, which cannot be accounted for by the Standard Model (SM), the observation of neutrino oscillations provides a strong motivation to search for new physics beyond the SM that are associated with neutrinos. Moreover, the existence of dark matter (DM) through abundant cosmological and astrophysical observations is one of the most plausible evidences of new physics beyond the SM. The DM direct detection experiments have pushed the limit on the cross section of DM scattering off nucleus close to the neutrino floor for weak scale DM. The couplings between the neutrinos and sub-GeV DM through the scattering off nucleus have been studied in refs. [2-9]. There are also plenty of novel models and signatures proposed to search for sub-GeV DM through the scattering off electrons [10-15].

Recently, the XENON collaboration reported an excess of electronic recoil events with the energy around $2-3 \mathrm{keV}[16]$ and the event distribution has a broad spectrum for the excess. They collected low energy electron recoil data from the XENON1T experiment with an exposure of 0.65 tonne-years and analyzed various backgrounds for the excess events. Although a small tritium background fits the excess data well, the solar axion explanation 
or the solar neutrinos with magnetic moment can also provide a plausible source for the peak-like excess. However, both of the two scenarios have tension with stellar cooling constraints [14, 17-21]. Some studies instead proposed to explain the XENON1T excess through the electron recoil by solar neutrinos with the sterile neutrino DM in the final states of inelastic scattering $[10,11]$. On the other hand, the inverse process in which the incoming fermionic DM is absorbed by bound electron targets and emits a neutrino is sensitive to the $\mathrm{DM}$ with mass below $\mathrm{MeV}[12,13]$. The two kinds of signals are governed by the same interactions between SM neutrino and the exotic fermion. The relevant interactions are inevitably constrained by the precision measurements in neutrino experiments $[14,15]$. In this work we study the constraints on general neutrino interactions with sub-GeV exotic fermion from neutrino-electron scattering experiments.

The large volume detectors enable precise measurements of neutrino properties. The large neutrino detectors like Borexino can be used to place constraints on general neutrino interactions. The Borexino experiment, located at the Laboratori Nazionali del Gran Sasso, was built with a primary goal of measuring solar neutrinos from the $p p$ chain [22]. We employ the Borexino measurements of low energy solar neutrinos to set limits on the neutrino-electron scattering with an outgoing fermion $\chi$

$$
\nu e \rightarrow \chi e,
$$

where $\chi$ could be sterile neutrino or other possible exotic fermions. The results apply for the exotic fermion $\chi$ being either DM candidate or not. We restrict the general neutrino interactions categorized by dimension- 5 dipole operators and dimension- 6 four-fermion operators. They respect Lorentz invariance and the gauge symmetries $\mathrm{SU}(3)_{c} \times \mathrm{U}(1)_{\mathrm{em}}$. The scattering cross section from the magnetic and electric dipole operators is inversely proportional to the recoil energy and thus the experiments with low energy threshold are sensitive to them. For the four-fermion interactions, all Lorentz-invariant operators (scalar, vector, pseudoscalar, axialvector and tensor) will be explored in the neutrino-electron scattering. As the produced solar electron neutrinos oscillate into muon and tau neutrinos, we can also place limits on the general interactions of all neutrino flavors. In addition, accelerator neutrinos with the energy being several tens of $\mathrm{GeV}$ can be used to exploit large $\chi$ mass region. We thus take into account the constraints from the CHARM-II experiment [23, 24] as well as reactor neutrino using TEXONO [25] data. The relevant data were used to study the electromagnetic interactions or the nonstandard vector-type interactions of a neutrino via the transition $\nu e \rightarrow \nu e$ [26-31]. The heavy neutral leptons as well as DM scattering off electrons can be also searched in high-energy neutrino beam-dump experiment or far-forward detector at the LHC [32, 33].

This paper is organized as follows. In section 2, we discuss the effective Lagrangian of an exotic fermion interacting with neutrino and electron. Then we display the amplitudes and differential cross sections of neutrino-electron scattering with the outgoing exotic fermion. In section 3, we consider three neutrino-electron scattering experiments: CHARM-II, TEXONO and Borexino, and show the constraints on the general neutrino interactions. The detection of sub-MeV DM absorbed by bound electron targets is then discussed in section 5. Finally, we summarize our conclusions in section 6 . 

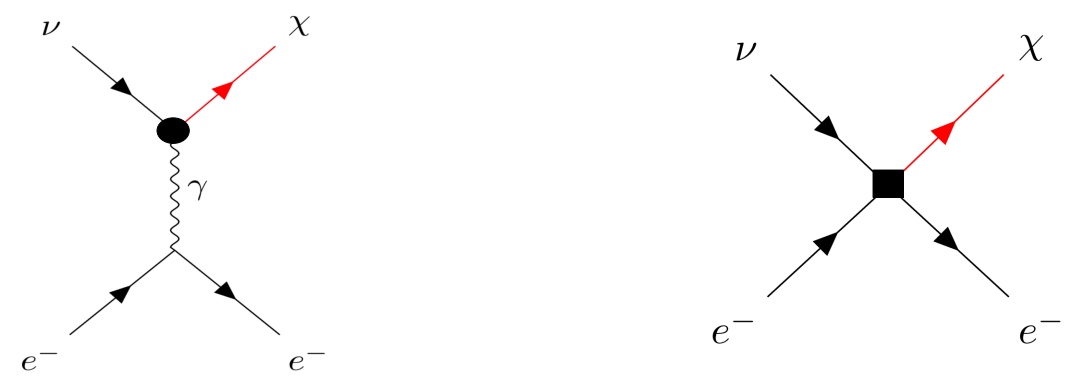

Figure 1. The tree-level Feynman diagrams for the $\nu_{\alpha}+e^{-} \rightarrow \chi+e^{-}$process, where the circular bulb (square) represents the effective dim- 5 dipole (dim-6 four-fermion) interaction.

\section{The general neutrino interactions with exotic fermion via neutrino- electron scattering}

We consider a Dirac fermion $\chi$ and its general interactions with neutrino and electron. The effective Lagrangian including both dim-5 dipole operators and dim- 6 four-fermion operators reads as

$$
\mathcal{L} \supset \frac{G_{F}}{\sqrt{2}}\left[\sum_{a} \bar{\chi} \Gamma^{a} \nu_{\alpha} \bar{e} \Gamma^{a}\left(\epsilon_{\alpha}^{a}+\tilde{\epsilon}_{\alpha}^{a} \gamma^{5}\right) e+\frac{v_{H}}{\sqrt{2}} \bar{\chi} \sigma^{\mu \nu}\left(\epsilon_{\alpha}^{M}+\epsilon_{\alpha}^{E} \gamma_{5}\right) \nu_{\alpha} F_{\mu \nu}\right]+\text { h.c. },
$$

where $\alpha \equiv\{e, \mu, \tau\}, v_{H} \simeq 246 \mathrm{GeV}$ is the vacuum expectation value of the SM Higgs, $F_{\mu \nu}$ is the electromagnetic field strength tensor and $\Gamma^{a} \equiv\left\{I, i \gamma^{5}, \gamma^{\mu}, \gamma^{\mu} \gamma^{5}, \sigma^{\mu \nu} \equiv \frac{i}{2}\left[\gamma^{\mu}, \gamma^{\nu}\right]\right\}$ correspond to the scalar $(S)$, pseudoscalar $(P)$, vector $(V)$, axialvector $(A)$ and tensor $(T)$ operator, respectively. The four-fermion operators are analogous to those in ref. [31]. Here the dimensionless parameters $\epsilon_{\alpha}^{M}, \epsilon_{\alpha}^{E}, \epsilon_{\alpha}^{a}$ and $\tilde{\epsilon}_{\alpha}^{a}$ are in general complex. The presence of new interactions of eq. (2.1) will give rise to the tree-level neutrino-electron scattering, as shown in figure 1.

In the SM, the neutrino-electron scattering is governed by both the weak neutral current (NC) and charged current (CC). The effective Lagrangian for the SM NC is given by

$$
\mathcal{L}_{\mathrm{NC}}=\frac{G_{F}}{\sqrt{2}} \bar{\nu} \gamma^{\mu}\left(1-\gamma_{5}\right) \nu \bar{e} \gamma_{\mu}\left(g_{V}-g_{A} \gamma_{5}\right) e,
$$

where $g_{V}=-\frac{1}{2}+2 \sin ^{2} \theta_{W}$ and $g_{A}=-\frac{1}{2}$. The CC Lagrangian can be transmitted as

$$
\mathcal{L}_{\mathrm{CC}}=\frac{G_{F}}{\sqrt{2}} \bar{\nu} \gamma^{\mu}\left(1-\gamma_{5}\right) \nu \bar{e} \gamma_{\mu}\left(1-\gamma_{5}\right) e .
$$

The CC current only contributes to the scattering of $\nu_{e}$. The differential cross section of neutrino-electron scattering in the SM is [31]

$$
\frac{d \sigma_{\alpha \beta}^{\mathrm{SM}}}{d E_{R}}=\frac{G_{F}^{2} m_{e}}{2 \pi}\left[\left(g_{\alpha \beta}^{L}\right)^{2}+\left(g_{\alpha \beta}^{R}\right)^{2}\left(1-\frac{E_{R}}{E_{\nu}}\right)^{2}-g_{\alpha \beta}^{L} g_{\alpha \beta}^{R} \frac{m_{e} E_{R}}{E_{\nu}^{2}}\right],
$$

where $\alpha(\beta)$ denotes the flavor of the neutrino in the initial (final) states, $E_{\nu}$ is the neutrino energy, $E_{R}$ is the electron recoil energy and

$$
\left(g_{\alpha \beta}^{L}, g_{\alpha \beta}^{R}\right)= \begin{cases}\left(2 \sin ^{2} \theta_{W}+1,2 \sin ^{2} \theta_{W}\right), & \alpha=\beta=e ; \\ \left(2 \sin ^{2} \theta_{W}-1,2 \sin ^{2} \theta_{W}\right), & \alpha=\beta=\mu, \tau \\ 0, & \alpha \neq \beta .\end{cases}
$$


We then calculate the differential cross section of $\nu_{\alpha}+e \rightarrow \chi+e$ by following the procedure given in the appendix. Here we show the differential cross sections for different operators:

$$
\begin{aligned}
\frac{d \sigma_{\nu_{\alpha}}^{S}}{d E_{R}}= & \frac{G_{F}^{2} m_{e}}{8 \pi}\left[\left|\epsilon_{\alpha}^{S}\right|^{2}\left(1+\frac{E_{R}}{2 m_{e}}\right)+\left|\tilde{\epsilon}_{\alpha}^{S}\right|^{2} \frac{E_{R}}{2 m_{e}}\right]\left(\frac{m_{e} E_{R}}{E_{\nu}^{2}}+\frac{m_{\chi}^{2}}{2 E_{\nu}^{2}}\right), \\
\frac{d \sigma_{\nu_{\alpha}}^{P}}{d E_{R}}= & \frac{G_{F}^{2} m_{e}}{8 \pi}\left[\left|\epsilon_{\alpha}^{P}\right|^{2} \frac{E_{R}}{2 m_{e}}+\left|\tilde{\epsilon}_{\alpha}^{P}\right|^{2}\left(1+\frac{E_{R}}{2 m_{e}}\right)\right]\left(\frac{m_{e} E_{R}}{E_{\nu}^{2}}+\frac{m_{\chi}^{2}}{2 E_{\nu}^{2}}\right), \\
\frac{d \sigma_{\nu_{\alpha}}^{V}}{d E_{R}}= & \frac{G_{F}^{2} m_{e}}{4 \pi}\left[\left(\left|\epsilon_{\alpha}^{V}\right|^{2}+\left|\tilde{\epsilon}_{\alpha}^{V}\right|^{2}\right)\left(1-\frac{E_{R}}{E_{\nu}}+\frac{E_{R}^{2}}{2 E_{\nu}^{2}}-\frac{m_{\chi}^{2}}{2 E_{\nu} m_{e}}+\frac{m_{\chi}^{2} E_{R}}{4 E_{\nu}^{2} m_{e}}\right)\right. \\
& \left.-\left(\left|\epsilon_{\alpha}^{V}\right|^{2}-\left|\tilde{\epsilon}_{\alpha}^{V}\right|^{2}\right)\left(\frac{E_{R} m_{e}}{2 E_{\nu}^{2}}+\frac{m_{\chi}^{2}}{4 E_{\nu}^{2}}\right)-2 \operatorname{Re}\left[\epsilon_{\alpha}^{V}\left(\tilde{\epsilon}_{\alpha}^{V}\right)^{*}\right] \frac{E_{R}}{E_{\nu}}\left(1-\frac{E_{R}}{2 E_{\nu}}-\frac{m_{\chi}^{2}}{4 E_{\nu} m_{e}}\right)\right], \\
\frac{d \sigma_{\nu_{\alpha}}^{A}}{d E_{R}}= & \frac{G_{F}^{2} m_{e}}{4 \pi}\left[\left(\left|\epsilon_{\alpha}^{A}\right|^{2}+\left|\tilde{\epsilon}_{\alpha}^{A}\right|^{2}\right)\left(1-\frac{E_{R}}{E_{\nu}}+\frac{E_{R}^{2}}{2 E_{\nu}^{2}}-\frac{m_{\chi}^{2}}{2 E_{\nu} m_{e}}+\frac{m_{\chi}^{2} E_{R}}{4 E_{\nu}^{2} m_{e}}\right)\right. \\
& \left.+\left(\left|\epsilon_{\alpha}^{A}\right|^{2}-\left|\tilde{\epsilon}_{\alpha}^{A}\right|^{2}\right)\left(\frac{E_{R} m_{e}}{2 E_{\nu}^{2}}+\frac{m_{\chi}^{2}}{4 E_{\nu}^{2}}\right)-2 \operatorname{Re}\left[\epsilon_{\alpha}^{A}\left(\tilde{\epsilon}_{\alpha}^{A}\right)^{*}\right] \frac{E_{R}}{E_{\nu}}\left(1-\frac{E_{R}}{2 E_{\nu}}-\frac{m_{\chi}^{2}}{4 E_{\nu} m_{e}}\right)\right], \\
\frac{d \sigma_{\nu_{\alpha}}^{T}}{d E_{R}}= & \frac{2 G_{F}^{2} m_{e}\left|\epsilon_{\alpha}^{T}-\tilde{\epsilon}_{\alpha}^{T}\right|^{2}}{\pi}\left[1-\frac{E_{R}}{E_{\nu}}+\frac{E_{R}^{2}}{4 E_{\nu}^{2}}-\frac{E_{R} m_{e}}{4 E_{\nu}^{2}}-\frac{m_{\chi}^{2}}{4 E_{\nu}^{2}}\left(\frac{1}{2}+\frac{2 E_{\nu}}{m_{e}}-\frac{E_{R}}{2 m_{e}}\right)\right],(2.10) \\
\frac{d \sigma_{\nu_{\alpha}}^{\mathrm{EM}}}{d E_{R}}= & \frac{2 \sqrt{2} \alpha_{\mathrm{EM}} G_{F}\left|\epsilon_{\alpha}^{E}-\epsilon_{\alpha}^{M}\right|^{2}}{m_{e}} \\
& \times\left[\frac{m_{e}}{E_{R}}-\frac{m_{e}}{E_{\nu}}-\frac{m_{\chi}^{2}}{E_{\nu} E_{R}}\left(\frac{1}{2}-\frac{E_{R}}{4 E_{\nu}}+\frac{m_{e}}{E_{\nu}}\right)-\frac{m_{\chi}^{4}}{8 E_{\nu}^{2} E_{R}^{2}}\left(1-\frac{E_{R}}{m_{e}}\right)\right],
\end{aligned}
$$

where $\alpha_{\mathrm{EM}} \simeq 1 / 137$ is the electromagnetic fine structure constant. Here we assume each of the scalar, pseudoscalar, vector, axialvector, tensor and electromagnetic dipole operators dominates at a time. The differential cross sections of $\bar{\nu}_{\alpha}+e \rightarrow \bar{\chi}+e$ are the same as those of $\nu_{\alpha}+e \rightarrow \chi+e$ except for the cross term of the axialvector operator in eq. (2.9) changed sign. From the above equations, we realize that the bounds on $\left\{\tilde{\epsilon}_{\alpha}^{S}, \tilde{\epsilon}_{\alpha}^{P}, \tilde{\epsilon}_{\alpha}^{V}, \tilde{\epsilon}_{\alpha}^{A}, \tilde{\epsilon}_{\alpha}^{T}, \epsilon_{\alpha}^{E}\right\}$ will be the same as $\left\{\epsilon_{\alpha}^{P}, \epsilon_{\alpha}^{S}, \epsilon_{\alpha}^{A}, \epsilon_{\alpha}^{V}, \epsilon_{\alpha}^{T}, \epsilon_{\alpha}^{M}\right\}$ if we consider only one $\epsilon$ parameter at a time.

\section{$3 \quad$ Neutrino-electron scattering experiments}

The parameter space of the general neutrino interactions with electrons can be constrained by various neutrino-electron scattering experiments such as CHARM-II [23, 24], LAMPF [34], LSND [35], TEXONO [25] and MINER $\nu \mathrm{A}[36,37]$. The precision of these neutrino-electron scattering experiments can be inferred from their measurement of $\sin ^{2} \theta_{W}$ [38], i.e., $\sin ^{2} \theta_{W}=0.2324 \pm 0.0083$ at CHARM-II, $0.249 \pm 0.063$ at LAMPF [34], $0.248 \pm 0.051$ at LSND [35], and $0.251 \pm 0.039$ at TEXONO [25]. Hence, we consider CHARM-II and TEXONO in our analysis since they have the strongest sensitivity to 

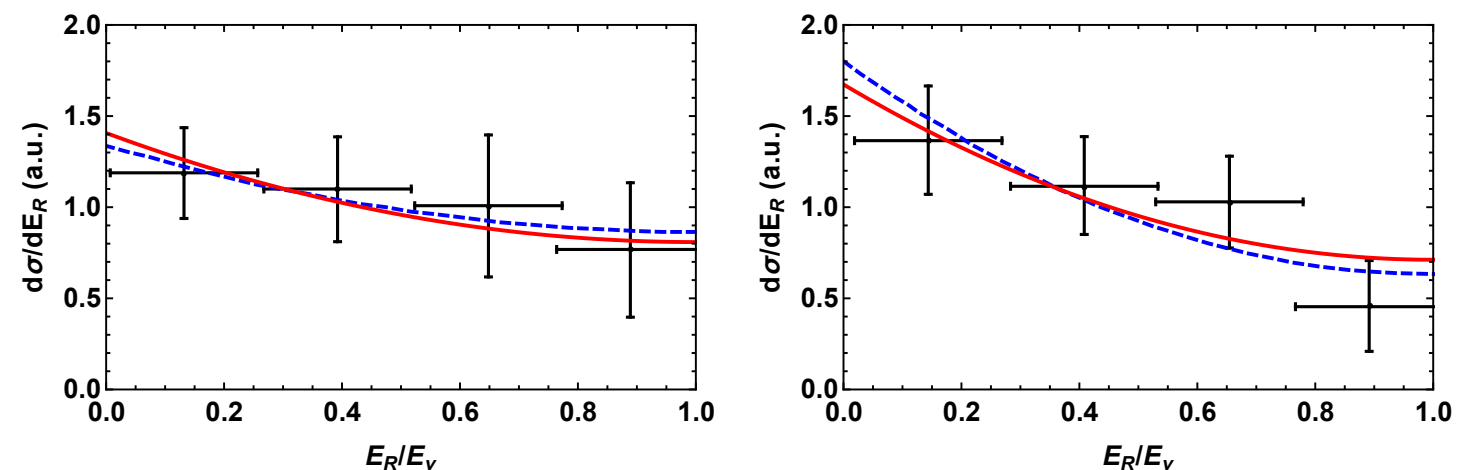

Figure 2. The differential cross sections of the $\nu_{\mu}$ (left) and $\bar{\nu}_{\mu}$ (right) scattering on electrons at CHARM-II. The black data points and the blue dashed SM prediction curve are taken from ref. [23]. The red curve corresponds to our best-fit prediction in the SM.

$\sin ^{2} \theta_{W} \cdot{ }^{1}$ We also consider the Borexino experiment since it measures the solar neutrinos which have much lower energies than other experiments.

\subsection{CHARM-II}

The CHARM-II experiments measured the high energy $\nu_{\mu}$ and $\bar{\nu}_{\mu}$ beam from the Super Proton Synchrotron (SPS) at CERN $[23,24]$. The mean neutrino energies of the $\nu_{\mu}$ and $\bar{\nu}_{\mu}$ beam are $23.7 \mathrm{GeV}$ and $19.1 \mathrm{GeV}$, respectively. The unfolded differential cross sections from the measurement have been given in ref. [23], and the data points are shown in figure 2. Our SM predictions are consistent with those given in the ref. [23]. Therefore, we consider the following $\chi^{2}$ function in our analysis for new physics:

$$
\chi_{\text {CHARM-II }}^{2}=\sum_{i} \frac{\left.\left(d \sigma / d E_{R}\right)_{i}-s_{i}^{0}\right)^{2}}{\sigma_{i}^{2}}+\left(\nu_{\mu} \rightarrow \bar{\nu}_{\mu}\right),
$$

where $s_{i}^{0}$ and $\sigma_{i}$ are the measured differential cross section and its corresponding uncertainties taken from ref. [23].

\subsection{TEXONO}

The cross section of $\bar{\nu}_{e}$ scattering on electrons has been measured by the TEXONO experiment utilizing electron antineutrinos produced by the Kuo-Sheng Nuclear Power Reactor with a $\mathrm{CsI}(\mathrm{Tl})$ scintillating crystal detector [25]. The detector is placed at a distance of 28 $\mathrm{m}$ from the $2.9 \mathrm{GW}$ reactor core. The range of recoil energy used in the analysis is from $3 \mathrm{MeV}$ and $8 \mathrm{MeV}$, respectively. The measured event rates and uncertainties have been given in ref. [25], which are shown in figure 3. As seen from the red and blue dashed curves in figure 3, our SM predictions agree quite well with those given in the ref. [25]. Therefore, we consider the following $\chi^{2}$ function in our analysis for new physics:

$$
\chi_{\mathrm{TEXONO}}^{2}=\sum_{i} \frac{\left(R_{i}^{0}-R_{i}(1+\alpha)\right)^{2}}{\sigma_{R, i}^{2}}+\left(\frac{\alpha}{\sigma_{\alpha}}\right)^{2},
$$

\footnotetext{
${ }^{1}$ The neutrino-electron scattering data at MINER $\nu \mathrm{A}$ can be also used to impose competitive bounds on new interactions as CHARM-II, see e.g. [39]. However, the full analysis of the measurement at MINER $\nu \mathrm{A}$ requires a dedicated Monte Carlo simulation of the sideband events, which is not available to us.
} 


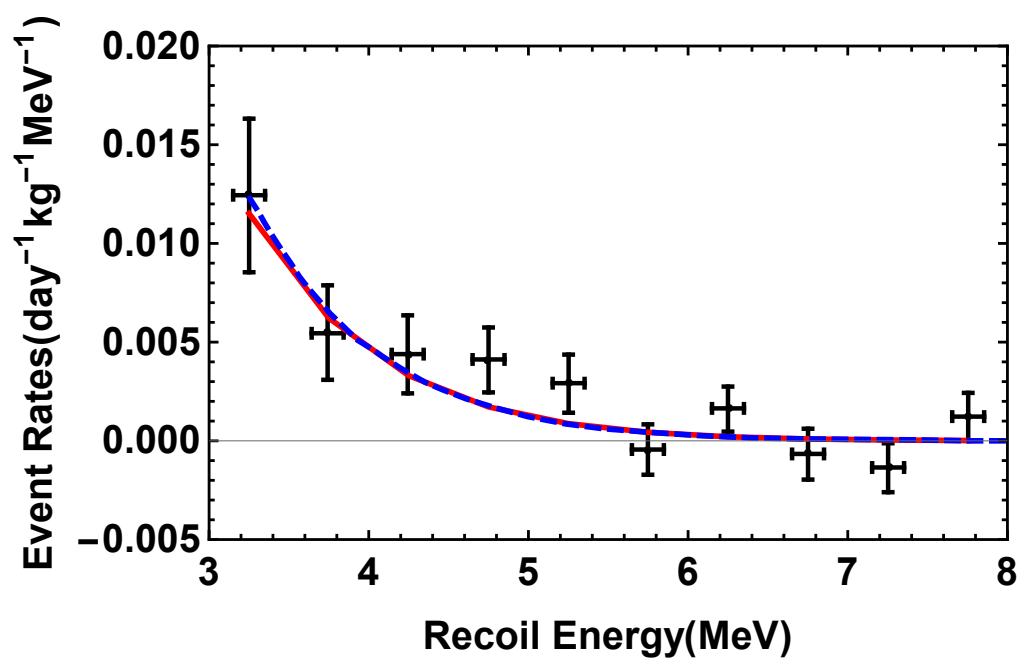

Figure 3. The event rates of $\bar{\nu}_{e}-e^{-}$scattering in the TEXONO experiment. The black data points and the blue dashed SM prediction curve are taken from ref. [25]. The red curve corresponds to our best-fit prediction in the SM.

where $R_{i}\left(R_{i}^{0}\right)$ and $\sigma_{R, i}$ are the predicted (measured) event rates and corresponding uncertainties in the $i$ th recoil energy bin. Here $\sigma_{\alpha}$ is the normalization uncertainty, and we take it to be $5 \%$ for conservation. Both the measured event rates and uncertainties are taken from ref. [25]. The predicted event rate in the $i$ th recoil energy bin is calculated by

$$
R_{i}=N_{e} \int d E_{\nu} \phi_{\bar{\nu}_{e}}\left(E_{\nu}\right) \int_{i} d E_{R} \frac{d \sigma_{\bar{\nu}_{e}}}{d E_{R}} \eta\left(E_{R}\right),
$$

where $N_{e}=2.5 \times 10^{26}$ is the number of target electrons in the CsI detector per kilogram, $\phi_{\bar{\nu}_{e}}\left(E_{\nu}\right)$ is the reactor antineutrino flux, and $\eta\left(E_{R}\right)$ is the detection efficiency. Here we take it to be $100 \%$, which yields a good agreement for the SM predictions shown in figure 16 of ref. [25].

\subsection{Borexino}

The Borexino experiment measured solar neutrinos at the Laboratori Nazionali del Gran Sasso. Only $\nu_{e}$ are produced in the core of the Sun. However, after adiabatic propagation in the sun, the solar neutrinos arrive at the Earth contain all three flavors: $\nu_{e}, \nu_{\mu}$ and $\nu_{\tau}$. The survival probability of solar neutrinos is given by [40]

$$
P_{e e} \approx s_{13}^{4}+c_{13}^{4}\left(c_{12}^{2} \cos ^{2} \theta_{12}^{m}+s_{12}^{2} \sin \theta_{12}^{m}\right),
$$

where $s_{12}\left(c_{12}\right)$ denotes $\sin \theta_{12}\left(\cos _{12}\right), \theta_{12}$ is the vacuum mixing angles in the PMNS matrix. Here $\theta_{12}^{m}$ is the effective mixing angle at the production point in the Sun, which is given by

$$
\theta_{12}^{m}=\frac{1}{2} \arctan \frac{\sin 2 \theta_{12}}{\cos 2 \theta_{12}-\hat{A} c_{13}^{2}},
$$

with $\hat{A} \equiv 2 \sqrt{2} G_{F} N_{e}^{S} E_{\nu} / \delta m_{21}^{2}$ and $N_{e}^{S}$ being the number density of electron at the production point in the Sun. Here we ignore the small corrections due to the day-night asymmetry 


\begin{tabular}{|c|c|c|c|}
\hline Source & Measurement $(\mathrm{cpd} / 100 \mathrm{t})$ & SM prediction $(\mathrm{cpd} / 100 \mathrm{t})$ & Percentage error \\
\hline$p p$ & $134 \pm 10_{-10}^{+6}$ & $136.0 \pm 1.6$ & $1.2 \%$ \\
\hline${ }^{7} B e$ & $46 \pm 1.5_{-1.6}^{+1.5}$ (phase I), $48.3 \pm 1.1_{-0.7}^{+0.4}$ (phase II) & $47.6 \pm 2.9$ & $6.1 \%$ \\
\hline$p e p$ & $3.1 \pm 0.6 \pm 0.3$ (phase I), $2.43 \pm 0.36_{-0.22}^{+0.15}$ (phase II) & $2.76 \pm 0.04$ & $1.3 \%$ \\
\hline${ }^{8} B$ & $0.223_{-0.016}^{+0.015} \pm 0.006$ & $0.209 \pm 0.025$ & $12.0 \%$ \\
\hline
\end{tabular}

Table 1. The measured event rates at Borexino and our predicted event rates in the SM. The theoretical percentage uncertainties are given in the last column.

in the Borexino measurement [41]. We consider the $p p,{ }^{7} \mathrm{Be}$ and pep spectra measured in the Borexino phase-I [42-44] and phase-II [22, 45], and the ${ }^{8} B$ data collected between January 2008 and December 2016 [46]. The expected event rate at Borexino is given by

$$
R_{\mathrm{pre}}^{i}=N_{e} \int d E_{\nu} \Phi^{i}\left(E_{\nu}\right)\left[P_{e e}^{i} \sigma_{e}\left(E_{\nu}\right)+\left(1-P_{e e}^{i}\right) \sigma_{\mu}\left(E_{\nu}\right)\right]
$$

where $N_{e}=3.307 \times 10^{31} / 100$ ton is the density of target electrons in the Borexino detector [22], and $i$ indicates solar neutrino sources $p p,{ }^{7} B e, p e p$ and ${ }^{8} B . \Phi^{i}\left(E_{\nu}\right)$ is the corresponding solar neutrino flux taken from the standard solar model (B16-GS98-HZ) [47]. $P_{e e}^{i}$ is the survival probability given in eq. (3.4). The cross section in eq. (3.6) is calculated by

$$
\sigma_{\alpha}=\int d E_{R} \frac{d \sigma_{\alpha}}{d E_{R}} \eta\left(E_{R}\right)
$$

where $\alpha=e, \mu, \frac{d \sigma_{\alpha}}{d E_{R}}$ is the differential cross section, and $\eta\left(E_{R}\right)$ is the detection efficiency. The detection efficiency for ${ }^{8} B$ is extracted from figure 2 in ref. [46] and we take $\eta\left(E_{R}\right)$ to be $100 \%$ for other solar neutrino sources [31]. The measured event rates and our predicted event rates in the SM are given in table 1 . We see that our SM predictions agree with the measured event rates. For new physics analysis, we employ the following $\chi^{2}$ function [31]:

$$
\chi_{\text {Borexino }}^{2}=\sum_{i} \frac{\left[R_{\text {exp }}^{i}-R_{\text {pre }}^{i}\left(1+\alpha_{i}\right)\right]^{2}}{\left(\sigma_{\text {stat }}^{i}\right)^{2}}+\left(\frac{\alpha^{i}}{\sigma_{\text {th }}^{i}}\right)^{2},
$$

where $R_{\exp }^{i}\left(\sigma_{\text {exp }}^{i}\right)$ are the central values (statistical uncertainties) of the $i$ th measurement given in table $1, R_{\text {pre }}^{i}$ is the predicted event rates calculated in eq. (3.6), and $\sigma_{t h}^{i}$ is the theoretical uncertainties given in the last column in table 1.

\section{Constraints from the experimental data}

In this section, we present our results of the constraints on the coupling coefficients of the general neutrino interactions with $\chi$ and electrons using the neutrino-electron scattering data from the CHARM-II, TEXONO and Borexino experiments.

\subsection{Flavor-universal bounds}

We firstly consider the flavor-universal couplings, i.e. by setting $\epsilon_{\alpha}^{i} \equiv \epsilon^{i}$ in eq. (2.1), where $i$ indicates the scalar $(\mathrm{S})$, pseudoscalar $(\mathrm{P})$, vector $(\mathrm{V})$, axialvector $(\mathrm{A})$, tensor $(\mathrm{T})$ and 
electromagnetic (E or $\mathrm{M}$ ) dipole operators. Here we also assume only one $\epsilon^{i}\left(\tilde{\epsilon}^{i}\right)$ exists at a time. As mentioned before, the bounds on $\left\{\tilde{\epsilon}_{\alpha}^{S}, \tilde{\epsilon}_{\alpha}^{P}, \tilde{\epsilon}_{\alpha}^{V}, \tilde{\epsilon}_{\alpha}^{A}, \tilde{\epsilon}_{\alpha}^{T}, \epsilon_{\alpha}^{E}\right\}$ will be the same as $\left\{\epsilon_{\alpha}^{P}, \epsilon_{\alpha}^{S}, \epsilon_{\alpha}^{A}, \epsilon_{\alpha}^{V}, \epsilon_{\alpha}^{T}, \epsilon_{\alpha}^{M}\right\}$ in this case. The $90 \%$ CL upper bounds on the magnitude of the coefficients $\epsilon^{i}\left(\tilde{\epsilon}^{i}\right)$ as a function of $m_{\chi}$ are shown in figure 4 . From figure 4, we see that the CHARM-II experiment yields the strongest bounds for the scalar, pseudoscalar, vector, axialvector and tensor interactions. For the electromagnetic dipole interaction, the Borexino experiment has the best sensitivity for $m_{\chi}$ below $1 \mathrm{MeV}$. There is an upper limit on $m_{\chi}$ for the CHARM-II bounds due to the kinematic constraint. This can be explained by eq. (A.6), from which we get $m_{\chi} \leq \sqrt{\left(2 E_{\nu}+m_{e}\right) m_{e}}-m_{e} \simeq 155 \mathrm{MeV}$ for $E_{\nu}=23.7 \mathrm{GeV}$. Also, the upper limits on $m_{\chi}$ from the TEXONO and Borexino experiments are much smaller than the CHARM-II experiment due to low neutrino energies used in these two experiments. In addition, we see that the bounds become flat at small $m_{\chi}$, which can be understood from eqs. (2.6), (2.7), (2.8), (2.9), (2.10) and (2.11) since the differential cross sections are insensitive to $m_{\chi}$ as $m_{\chi} \ll E_{\nu}$. From figure 4 , we find that for $m_{\chi} \lesssim 50 \mathrm{MeV}$, the strongest bounds on the magnitude of $\epsilon^{S, P}\left(\tilde{\epsilon}^{S, P}\right), \epsilon^{V, A}\left(\tilde{\epsilon}^{V, A}\right)$ and $\epsilon^{T}\left(\tilde{\epsilon}^{T}\right)$ can reach $1.0,0.5$ and 0.2 , respectively. The strongest bounds on the magnitude of $\epsilon^{M, E}$ can reach $1.3 \times 10^{-6}$ for $m_{\chi} \lesssim 0.1 \mathrm{MeV}$.

\subsection{Flavor-dependent bounds}

Since only $\nu_{\mu}\left(\bar{\nu}_{\mu}\right)$ are measured at the CHARM-II experiment and only $\bar{\nu}_{e}$ are measured at the TEXONO experiment, the bounds from these two experiments can be avoided if the coupling coefficients are flavor non-universal. To illustrate the flavor dependence of these bounds, we show the $90 \%$ CL allowed regions in the $\left(\epsilon_{e}, \epsilon_{\mu}\right)$ plane for the scalar and vector interactions in figure 5. Here we fixed $m_{\chi}=1 \mathrm{MeV}$, and assume the coupling coefficients are real for simplicity. We also assume $\epsilon_{\tau}=\epsilon_{\mu}$ for the Borexino experiments. As seen from figure 5, the CHARM-II experiment is not sensitive to $\epsilon_{e}$, and the TEXONO experiment has no sensitivity to $\epsilon_{\mu}$. The solar neutrino experiment at Borexino can impose constraints on both $\epsilon_{e}$ and $\epsilon_{\mu}$ due to the flavor transition in the Sun. We also show the allowed regions of the combined data from these three experiments as the gray shaded regions in figure 5 . From figure 5, one can see that the sensitivity of the combined data mainly comes from the CHARM-II and TEXONO experiment.

\section{Prospects at DM experiments}

The exotic fermion $\chi$ can also serve as a DM particle if the correct DM abundance is satisfied. The relic abundance of DM is model dependent and changes based on the specific production mechanism and dynamics in the early Universe. The DM is either in thermal equilibrium with the SM bath and freezes out below some temperature, or is produced non-thermally. The thermal production of DM is a well-studied mechanism to obtain the correct abundance of DM in the early Universe. In our case, DM starts to keep in thermal equilibrium with the SM bath as a result of both the forward and backward processes $\bar{\chi} \chi \leftrightarrow \bar{\nu} \nu$ happening in the early Universe. As the Universe expands, the temperature drops below the DM mass and the DM falls out of thermal equilibrium. As a result, 

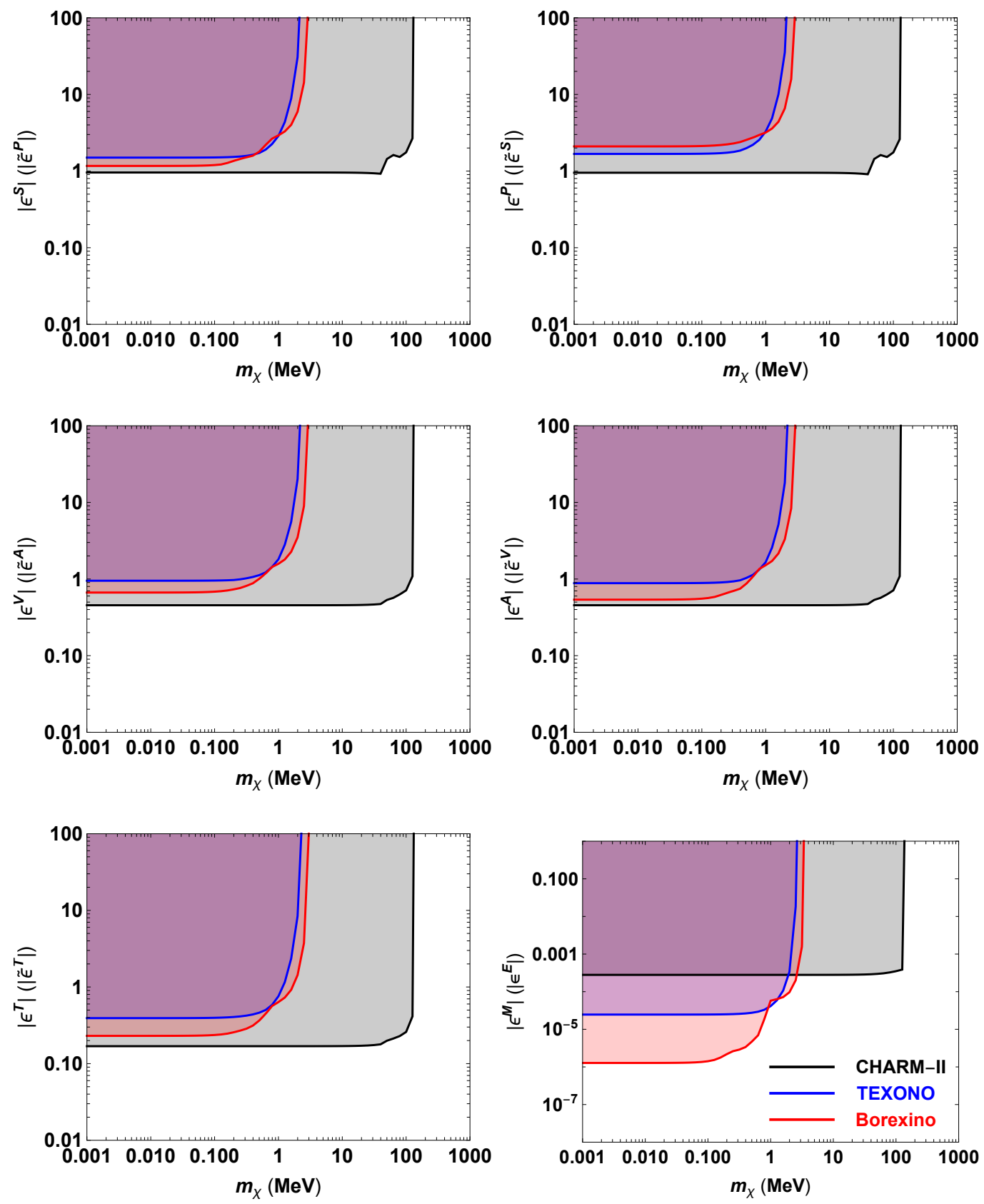

Figure 4. The $90 \%$ CL upper bounds on the magnitude of coupling coefficients as a function of $m_{\chi}$ for the scalar, pseudoscalar, vector, axialvector, tensor and electromagnetic dipole interactions. We assume that the couplings are flavor universal and only one $\epsilon^{i}\left(\tilde{\epsilon}^{i}\right)$ exists at a time. The gray, blue and red shaded regions are excluded by the CHARM-II, TEXONO and Borexino experiment, respectively. The bounds on $\left|\tilde{\epsilon}_{\alpha}^{S}\right|,\left|\tilde{\epsilon}_{\alpha}^{P}\right|,\left|\tilde{\epsilon}_{\alpha}^{V}\right|,\left|\tilde{\epsilon}_{\alpha}^{A}\right|,\left|\tilde{\epsilon}_{\alpha}^{T}\right|$, and $\left|\epsilon_{\alpha}^{E}\right|$ are the same as $\left|\epsilon_{\alpha}^{P}\right|,\left|\epsilon_{\alpha}^{S}\right|,\left|\epsilon_{\alpha}^{A}\right|,\left|\epsilon_{\alpha}^{V}\right|,\left|\epsilon_{\alpha}^{T}\right|$, and $\left|\epsilon_{\alpha}^{M}\right|$, respectively. 

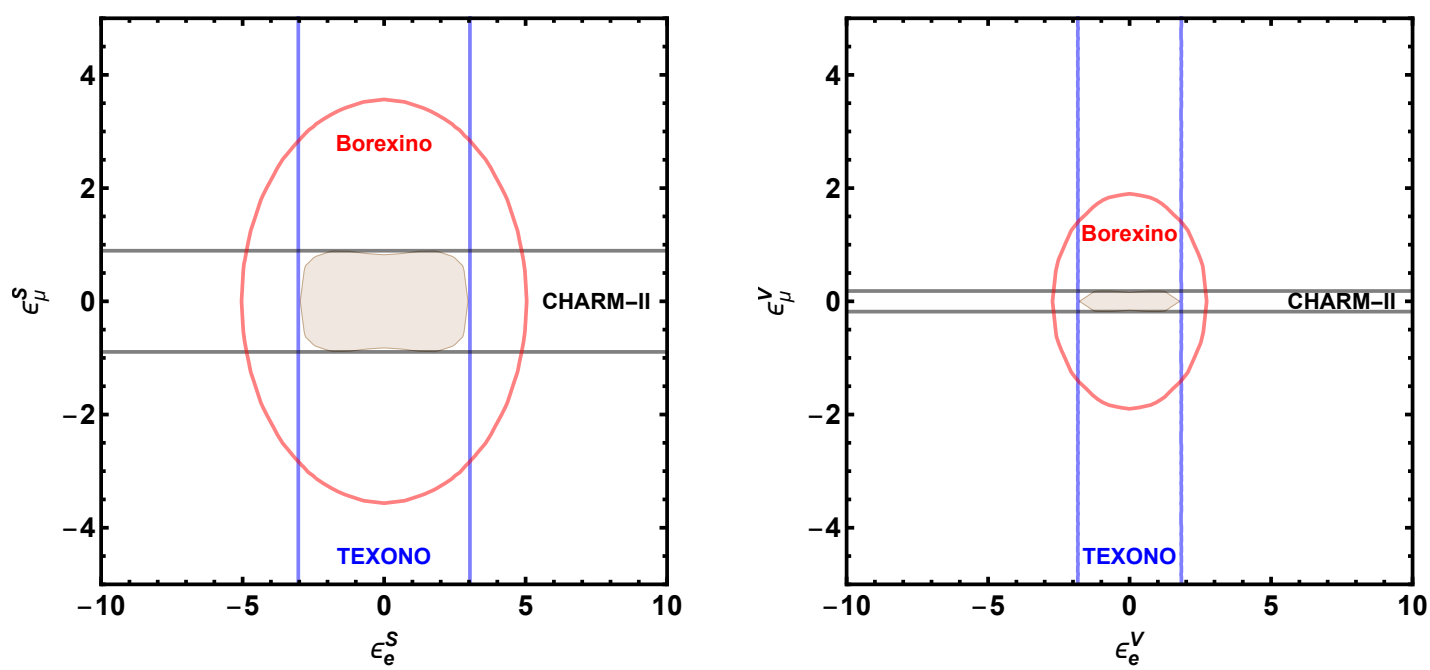

Figure 5. The 90\% CL allowed regions in the $\left(\epsilon_{e}, \epsilon_{\mu}\right)$ plane for the scalar (left panel) and vector (right panel) interactions. Here we assume $m_{\chi}=1 \mathrm{MeV}$ and the coupling coefficients are real with $\epsilon_{\tau}=\epsilon_{\mu}$. The region enclosed by the black, blue and red curves correspond to the CHARM-II, TEXONO and Borexino experiments, respectively. The gray shaded regions correspond to the allowed regions of the combined data from these three experiments.

only the forward process of the annihilation of DM particles into neutrinos occurs and the abundance of DM is quickly suppressed so as to reach the current equilibrium abundance. This is the so-called freeze-out process for the production of DM. The DM in thermal equilibrium should not significantly modifies the standard predictions of the ratios of light elemental abundances by the big bang nucleosynthesis (BBN). Also, the thermal DM species must not significantly alter the temperature ratio of photons and neutrinos at recombination. It turns out that thermal $\mathrm{DM}$ lighter than $\mathrm{MeV}$ scale will contribute to the radiation energy density and be ruled out by $N_{\text {eff }}$ constraint [48]. For the DM mass scale smaller than $\mathrm{MeV}$, the above $\chi-\nu$ operators can induce the non-thermal production of DM after the SM neutrinos decouple and before the electrons leave the bath. In the case of non-thermal production, if the coupling to electron is large, the DM would suffer the constraint from overproduction near BBN. The calculation of the final DM abundance replies on the details of the UV completion. The annihilation cross section depends on the $\chi-\nu$ mixing and the nature of mediator such as a dark photon for vector interaction or a scalar for scalar interaction in a UV completion. We refer the UV models to ref. [13] and one can see that the XENON1T excess preferred parameter space can avoid overproducing the DM abundance.

Here we briefly discuss the detection of DM hypothesis and the XENON1T excess. If we reverse the above process and interpret the exotic fermion $\chi$ as DM particle, the incoming DM $\chi$ can be absorbed by bound electron targets and emit a neutrino

$$
\chi e \rightarrow \nu e .
$$

For the DM elastic scattering off the electron, to explain the XENON1T excess, the key point is how to produce abundant DM particles with high velocity $v_{\mathrm{DM}} \gtrsim 0.1$ [49]. In 
contrast, ref. [13] proposed the above DM absorption scenario in which a DM particle deposits its mass energy rather than kinetic energy and it is sensitive to sub-MeV fermionic DM. For the DM absorption with free electrons $\chi e \rightarrow \nu e$, analogous to the case with nucleus absorbing the DM $[4,5]$, the total event rate is naively given by

$$
R=\frac{\rho_{\chi}}{m_{\chi}} \sigma_{e} N_{T} \Theta\left(E_{R}^{0}-E_{\mathrm{th}}\right),
$$

where $N_{T}$ is the number of target nuclei per detector mass, the local DM density is $\rho_{\chi} \simeq$ $0.4 \mathrm{GeV} / \mathrm{cm}^{3}, \Theta$ is the Heaviside theta function, $E_{\mathrm{th}}$ is the experimental threshold, $E_{R}^{0}=$ $m_{\chi}^{2} / 2 m_{e}$ for a free electron absorbing the DM, and $\sigma_{e}$ is the absorption cross section per electron. For the XENON1T experiment, we have $N_{T} \simeq 4 \times 10^{27} /$ tonne and $E_{R}^{0} \simeq 2 \mathrm{keV}$ giving $m_{\chi} \simeq 45 \mathrm{keV}$. With the total exposure being 0.65 tonne.years, XENON1T observed 285 events and the expected event number is $232 \pm 15$. This gives the total scattering cross section $\sigma_{e} \simeq 2 \times 10^{-48} \mathrm{~cm}^{2}$. In fact, the absorbing electron in a shell with a binding energy would be ionized with recoil energy [50, 51]. In eq. (5.2) there should also appear the ionization form factor of an electron in a certain shell and the total differential ionization rate is obtained by summing over all possible shells of the absorbing target electrons. The recoil energy of a free electron absorbing the DM $E_{R}^{0}$ is then shifted. The best fit to the XENON1T data was found to be $\left(m_{\chi}=56.5 \mathrm{keV}, \sigma_{e}=1 \times 10^{-49} \mathrm{~cm}^{2}\right)$ [13]. We take the vector interaction for illustration. The total scattering cross section $\sigma_{e}$ for vector operators is

$$
\sigma_{e}^{V}=\frac{G_{F}^{2} m_{\chi}^{2}\left(m_{\chi}+2 m_{e}\right)^{2}}{32 \pi\left(m_{e}+m_{\chi}\right)^{4}}\left[\left|\epsilon^{V}\right|^{2}\left(2 m_{e}^{2}+4 m_{e} m_{\chi}+3 m_{\chi}^{2}\right)+\left|\tilde{\epsilon}^{V}\right|^{2}\left(6 m_{e}^{2}+8 m_{e} m_{\chi}+3 m_{\chi}^{2}\right)\right] .
$$

By transforming the above best fit to the parameterization in our context, one essentially obtains $\left|\epsilon^{V}\right|,\left|\tilde{\epsilon}^{V}\right| \simeq 0.1$ which is allowed by the neutrino scattering experiments. Note that the LUX-ZEPLIN (LZ) experiment at the Sanford Underground Research Facility (SURF) in South Dakota [52] will start to take data soon and is able to probe orders of magnitude more parameter space than XENON1T. Such next generation detector will help to test the XENON1T favored region.

The decaying DM scenario usually faces the requirement of stability. The corresponding lifetime of $\chi$ should be longer than the age of the Universe, i.e. $t_{\text {Universe }}=$ $4.4 \times 10^{17} \mathrm{sec}[53]$. Requiring the DM being stable at the Universe time scale would set a very stringent bound on the coupling and/or the DM mass. For the vector interaction in our effective framework, the leading decay process is $\chi \rightarrow \nu \gamma$ with one photon radiated from the closed electron loop. The constraint would be quite stringent if only electron is involved in the calculation of the above decay width. In a realistic UV model in ref. [13] with $\chi$ only coupled to right-handed neutrino, the decay $\chi \rightarrow \nu \gamma$ is suppressed by the insertion of neutrino mass. The decay of $\chi$ into $\nu \gamma \gamma$ is forbidden and the leading decay becomes $\chi \rightarrow \nu \gamma \gamma \gamma$ which leads to a quite weak constraint. We refer the detailed discussion of the sub-MeV DM absorption by electrons to ref. [13] and future studies. On the other hand, more stringent constraints can also be placed on decaying DM from the observations of the galactic and extra-galactic diffuse X-ray or gamma-ray background. For 
several tens of keV DM, strong constraints have been obtained using INTEGRAL [54-56] and NuSTAR $[57,58]$. The bound on the lifetime of decaying DM depends on the specific model parameters and decay topologies.

\section{Conclusion}

In this work we study the constraints on general neutrino interactions with sub-GeV exotic fermion $\chi$ from neutrino-electron scattering experiments. The general neutrino interactions are composed of dimension-5 dipole operators and dimension- 6 four-fermion operators. We employ the measurements of CHARM-II, TEXONO and Borexino experiments to set limits on the neutrino-electron scattering with an outgoing fermion $\chi$. We find that the bounds are dominated by the CHARM-II experiment in most of the parameter space for the flavor-universal interactions and $m_{\chi}$ below $155 \mathrm{MeV}$, while the Borexino experiment sets the strongest bounds in the low mass region for the electromagnetic dipole interactions. The limits are found to be $\left|\epsilon^{S, P}\right|\left(\left|\tilde{\epsilon}^{S, P}\right|\right)<1,\left|\epsilon^{V, A}\right|\left(\left|\tilde{\epsilon}^{V, A}\right|\right)<0.5,\left|\epsilon^{T}\right|\left(\left|\tilde{\epsilon}^{T}\right|\right)<0.2$ for $m_{\chi} \lesssim 50 \mathrm{MeV}$ and $\left|\epsilon^{M, E}\right|<1.3 \times 10^{-6}$ for $m_{\chi} \lesssim 0.1 \mathrm{MeV}$. If the coupling coefficients are flavor non-universal, the bounds on $\epsilon_{e}\left(\epsilon_{\mu}\right)$ can be avoided for the CHARM-II (TEXONO) experiment, and there are correlations between the bounds on the coupling coefficients from the Borexino experiment. Finally, as an example, we discuss the detection of sub-MeV DM absorbed by bound electron targets. By transforming the best fit to the XENON1T data in our parameterization, we obtain the preferred coefficients for vector interactions as $\left|\epsilon^{V}\right|,\left|\tilde{\epsilon}^{V}\right| \simeq 0.1$ which is allowed by the neutrino experiments.

\section{Acknowledgments}

TL is supported by the National Natural Science Foundation of China (Grant No. 12035008, 11975129) and "the Fundamental Research Funds for the Central Universities", Nankai University (Grant No. 63196013). JL is supported by the National Natural Science Foundation of China (Grant No. 11905299), Guangdong Basic and Applied Basic Research Foundation (Grant No. 2020A1515011479), the Fundamental Research Funds for the Central Universities, and the Sun Yat-Sen University Science Foundation.

\section{A Calculation of the differential cross section}

The amplitude for $\nu\left(p_{1}\right) e\left(k_{1}\right) \rightarrow \chi\left(p_{2}\right) e\left(k_{2}\right)$ is given by

$$
\begin{aligned}
\mathcal{M}= & \frac{G_{F}}{\sqrt{2}} \bar{u}_{\chi}\left(p_{2}\right) P_{L} u_{\nu}\left(p_{1}\right) \bar{u}_{e}\left(k_{2}\right)\left(\epsilon^{S}+\tilde{\epsilon}^{S} \gamma_{5}\right) u_{e}\left(k_{1}\right) \\
& +\frac{G_{F}}{\sqrt{2}} \bar{u}_{\chi}\left(p_{2}\right) i \gamma_{5} P_{L} u_{\nu}\left(p_{1}\right) \bar{u}_{e}\left(k_{2}\right) i \gamma_{5}\left(\epsilon^{P}+\tilde{\epsilon}^{P} \gamma_{5}\right) u_{e}\left(k_{1}\right) \\
& +\frac{G_{F}}{\sqrt{2}} \bar{u}_{\chi}\left(p_{2}\right) \gamma_{\mu} P_{L} u_{\nu}\left(p_{1}\right) \bar{u}_{e}\left(k_{2}\right) \gamma^{\mu}\left(\epsilon^{V}+\tilde{\epsilon}^{V} \gamma_{5}\right) u_{e}\left(k_{1}\right) \\
& +\frac{G_{F}}{\sqrt{2}} \bar{u}_{\chi}\left(p_{2}\right) \gamma_{\mu} \gamma_{5} P_{L} u_{\nu}\left(p_{1}\right) \bar{u}_{e}\left(k_{2}\right) \gamma^{\mu} \gamma_{5}\left(\epsilon^{A}+\tilde{\epsilon}^{A} \gamma_{5}\right) u_{e}\left(k_{1}\right)
\end{aligned}
$$




$$
\begin{aligned}
& +\frac{G_{F}}{\sqrt{2}} \bar{u}_{\chi}\left(p_{2}\right) \sigma_{\mu \nu} P_{L} u_{\nu}\left(p_{1}\right) \bar{u}_{e}\left(k_{2}\right) \sigma^{\mu \nu}\left(\epsilon^{T}+\tilde{\epsilon}^{T} \gamma_{5}\right) u_{e}\left(k_{1}\right) \\
& +\frac{i G_{F} v_{H} e Q_{e}}{t^{2}} \bar{u}_{\chi}\left(p_{2}\right) \sigma_{\mu \nu}\left(\epsilon^{M}+\epsilon^{E} \gamma_{5}\right) P_{L} u_{\nu}\left(p_{1}\right) \bar{u}_{e}\left(k_{2}\right) \gamma_{\mu} t_{\nu} u_{e}\left(k_{1}\right),
\end{aligned}
$$

where the projector $P_{L}=\left(1-\gamma_{5}\right) / 2$ is inserted to force the incoming neutrinos to be left-handed and $t=p_{1}-p_{2}$. The amplitude for $\bar{\nu}\left(p_{1}\right) e\left(k_{1}\right) \rightarrow \bar{\chi}\left(p_{2}\right) e\left(k_{2}\right)$ is given by

$$
\begin{aligned}
\mathcal{M}= & \frac{G_{F}}{\sqrt{2}} \bar{v}_{\nu}\left(p_{1}\right) P_{R} v_{\chi}\left(p_{2}\right) \bar{u}_{e}\left(k_{2}\right)\left(\epsilon^{S *}-\tilde{\epsilon}^{S *} \gamma_{5}\right) u_{e}\left(k_{1}\right) \\
& +\frac{G_{F}}{\sqrt{2}} \bar{v}_{\nu}\left(p_{1}\right) P_{R} i \gamma_{5} v_{\chi}\left(p_{2}\right) \bar{u}_{e}\left(k_{2}\right) i \gamma_{5}\left(\epsilon^{P *}-\tilde{\epsilon}^{P *} \gamma_{5}\right) u_{e}\left(k_{1}\right) \\
& +\frac{G_{F}}{\sqrt{2}} \bar{v}_{\nu}\left(p_{1}\right) P_{R} \gamma_{\mu} v_{\chi}\left(p_{2}\right) \bar{u}_{e}\left(k_{2}\right) \gamma^{\mu}\left(\epsilon^{V *}+\tilde{\epsilon}^{V *} \gamma_{5}\right) u_{e}\left(k_{1}\right) \\
& +\frac{G_{F}}{\sqrt{2}} \bar{v}_{\nu}\left(p_{1}\right) P_{R} \gamma_{\mu} \gamma_{5} v_{\chi}\left(p_{2}\right) \bar{u}_{e}\left(k_{2}\right) \gamma^{\mu} \gamma_{5}\left(\epsilon^{A *}+\tilde{\epsilon}^{A *} \gamma_{5}\right) u_{e}\left(k_{1}\right) \\
& +\frac{G_{F}}{\sqrt{2}} \bar{v}_{\nu}\left(p_{1}\right) P_{R} \sigma_{\mu \nu} v_{\chi}\left(p_{2}\right) \bar{u}_{e}\left(k_{2}\right) \sigma^{\mu \nu}\left(\epsilon^{T *}-\tilde{\epsilon}^{T *} \gamma_{5}\right) u_{e}\left(k_{1}\right) \\
& +\frac{i G_{F} v_{H} e Q_{e}}{t^{2}} \bar{v}_{\nu}\left(p_{1}\right) P_{R} \sigma_{\mu \nu}\left(\epsilon^{M *}-\epsilon^{E *} \gamma_{5}\right) v_{\chi}\left(p_{2}\right) \bar{u}_{e}\left(k_{2}\right) \gamma_{\mu} t_{\nu} u_{e}\left(k_{1}\right)
\end{aligned}
$$

where the projector $P_{R}=\left(1+\gamma_{5}\right) / 2$ is inserted to force the incoming anti-neutrinos to be right-handed. The differential cross section of neutrino-electron scattering $\nu(\bar{\nu})+e \rightarrow$ $\chi(\bar{\chi})+e$ is

$$
\frac{d \sigma(\nu e)}{d E_{R}}=\frac{1}{32 \pi m_{e} E_{\nu}^{2}} \overline{|\mathcal{M}|^{2}}
$$

where $\overline{|\mathcal{M}|^{2}}$ is the spin-averaged amplitude square. The scattering angle is

$$
\cos \theta=\frac{E_{R}\left(E_{\nu}+m_{e}\right)+m_{\chi}^{2} / 2}{E_{\nu} \sqrt{E_{R}^{2}+2 m_{e} E_{R}}} .
$$

By requiring $\cos \theta \leq 1$, we can get the bounds on $E_{R}$ as

$$
E_{R}^{\min (\max )}=\frac{2 m_{e} E_{\nu}^{2}-m_{\chi}^{2}\left(m_{e}+E_{\nu}\right) \mp E_{\nu} \sqrt{\left(2 m_{e} E_{\nu}-m_{\chi}^{2}\right)^{2}-4 m_{e}^{2} m_{\chi}^{2}}}{2 m_{e}\left(m_{e}+2 E_{\nu}\right)}
$$

and the minimal energy to generate the elastic scattering is

$$
E_{\nu}^{\min }=m_{\chi}+\frac{m_{\chi}^{2}}{2 m_{e}}
$$

Open Access. This article is distributed under the terms of the Creative Commons Attribution License (CC-BY 4.0), which permits any use, distribution and reproduction in any medium, provided the original author(s) and source are credited. 


\section{References}

[1] Particle Data Group collaboration, Review of particle physics, PTEP 2020 (2020) $083 \mathrm{C} 01$ [INSPIRE].

[2] V. Brdar, W. Rodejohann and X.-J. Xu, Producing a new Fermion in coherent elastic neutrino-nucleus scattering: from neutrino mass to dark matter, JHEP 12 (2018) 024 [arXiv: 1810.03626] [INSPIRE].

[3] W.-F. Chang and J.N. Ng, KeV scale new fermion from a hidden sector, Phys. Rev. D 101 (2020) 035028 [arXiv: 1903.12545] [INSPIRE].

[4] J.A. Dror, G. Elor and R. Mcgehee, Directly detecting signals from absorption of fermionic dark matter, Phys. Rev. Lett. 124 (2020) 18 [arXiv: 1905.12635] [INSPIRE].

[5] J.A. Dror, G. Elor and R. Mcgehee, Absorption of fermionic dark matter by nuclear targets, JHEP 02 (2020) 134 [arXiv: 1908.10861] [INSPIRE].

[6] W.-F. Chang and J. Liao, Constraints on light singlet fermion interactions from coherent elastic neutrino-nucleus scattering, Phys. Rev. D 102 (2020) 075004 [arXiv:2002.10275] [INSPIRE].

[7] T. Li, X.-D. Ma and M.A. Schmidt, General neutrino interactions with sterile neutrinos in light of coherent neutrino-nucleus scattering and meson invisible decays, JHEP 07 (2020) 152 [arXiv:2005.01543] [INSPIRE].

[8] N. Hurtado, H. Mir, I.M. Shoemaker, E. Welch and J. Wyenberg, Dark matter-neutrino interconversion at COHERENT, direct detection, and the early universe, Phys. Rev. D 102 (2020) 015006 [arXiv:2005.13384] [INSPIRE].

[9] T. Li and J. Liao, Loop effect in the coherent neutrino-nucleus scattering, JHEP 02 (2021) 099 [arXiv:2008.00743] [INSPIRE].

[10] S.F. Ge, P. Pasquini and J. Sheng, Solar neutrino scattering with electron into massive sterile neutrino, Phys. Lett. B 810 (2020) 135787 [arXiv:2006.16069].

[11] I.M. Shoemaker, Y.-D. Tsai and J. Wyenberg, An active-to-sterile neutrino transition dipole moment and the XENON1T excess, arXiv:2007.05513 [INSPIRE].

[12] S. Shakeri, F. Hajkarim and S.-S. Xue, Shedding new light on sterile neutrinos from XENON1T experiment, JHEP 12 (2020) 194 [arXiv:2008.05029] [INSPIRE].

[13] J.A. Dror, G. Elor, R. McGehee and T.-T. Yu, Absorption of sub-MeV fermionic dark matter by electron targets, Phys. Rev. D 103 (2021) 035001 [arXiv:2011.01940] [InSPIRE].

[14] V. Brdar, A. Greljo, J. Kopp and T. Opferkuch, The neutrino magnetic moment portal: cosmology, astrophysics, and direct detection, JCAP 01 (2021) 039 [arXiv:2007.15563] [INSPIRE].

[15] D. Aristizabal Sierra, R. Branada, O.G. Miranda and G. Sanchez Garcia, Sensitivity of direct detection experiments to neutrino magnetic dipole moments, JHEP 12 (2020) 178 [arXiv:2008.05080] [INSPIRE].

[16] XENON collaboration, Excess electronic recoil events in XENON1T, Phys. Rev. D 102 (2020) 072004 [arXiv:2006.09721] [INSPIRE].

[17] N. Viaux et al., Neutrino and axion bounds from the globular cluster M5 (NGC 5904), Phys. Rev. Lett. 111 (2013) 231301 [arXiv:1311.1669] [InSPIRE].

[18] S.A. Díaz, K.-P. Schröder, K. Zuber, D. Jack and E.E.B. Barrios, Constraint on the axion-electron coupling constant and the neutrino magnetic dipole moment by using the tip-RGB luminosity of fifty globular clusters, arXiv:1910.10568 [INSPIRE]. 
[19] L. Di Luzio, M. Fedele, M. Giannotti, F. Mescia and E. Nardi, Solar axions cannot explain the XENON1T excess, Phys. Rev. Lett. 125 (2020) 131804 [arXiv: 2006.12487] [InSPIRE].

[20] C. Gao, J. Liu, L.-T. Wang, X.-P. Wang, W. Xue and Y.-M. Zhong, Reexamining the solar axion explanation for the XENON1T excess, Phys. Rev. Lett. 125 (2020) 131806 [arXiv:2006.14598] [INSPIRE].

[21] J.B. Dent, B. Dutta, J.L. Newstead and A. Thompson, Inverse Primakoff scattering as a probe of solar axions at liquid xenon direct detection experiments, Phys. Rev. Lett. 125 (2020) 131805 [arXiv:2006.15118] [INSPIRE].

[22] BoreXiNo collaboration, First simultaneous precision spectroscopy of pp ${ }^{7}$ Be, and pep solar neutrinos with Borexino Phase-II, Phys. Rev. D 100 (2019) 082004 [arXiv:1707.09279] [INSPIRE].

[23] CHARM-II collaboration, Measurement of differential cross-sections for muon-neutrino electron scattering, Phys. Lett. B 302 (1993) 351 [INSPIRE].

[24] CHARM-II collaboration, Precision measurement of electroweak parameters from the scattering of muon-neutrinos on electrons, Phys. Lett. B 335 (1994) 246 [INSPIRE].

[25] TEXONO collaboration, Measurement of $\bar{\nu}_{e}$-e scattering cross-section with a CsI(Tl) scintillating crystal array at the Kuo-Sheng nuclear power reactor, Phys. Rev. D 81 (2010) 072001 [arXiv: 0911.1597] [INSPIRE].

[26] A.N. Khan, D.W. McKay and F. Tahir, Short baseline reactor $\bar{\nu}-e$ scattering experiments and nonstandard neutrino interactions at source and detector, Phys. Rev. D 90 (2014) 053008 [arXiv: 1407.4263] [INSPIRE].

[27] A.N. Khan, Global analysis of the source and detector nonstandard interactions using the short baseline $\nu$-e and $\nu$-e scattering data, Phys. Rev. D 93 (2016) 093019 [arXiv: 1605.09284] [INSPIRE].

[28] A.N. Khan and D.W. McKay, $\sin ^{2}(\theta) w$ estimate and bounds on nonstandard interactions at source and detector in the solar neutrino low-energy regime, JHEP 07 (2017) 143 [arXiv: 1704.06222] [INSPIRE].

[29] A.N. Khan, $\sin ^{2} \theta_{W}$ estimate and neutrino electromagnetic properties from low-energy solar data, J. Phys. G 46 (2019) 035005 [arXiv:1709. 02930] [inSPIRE].

[30] M. Lindner, F.S. Queiroz, W. Rodejohann and X.-J. Xu, Neutrino-electron scattering: general constraints on $Z^{\prime}$ and dark photon models, JHEP 05 (2018) 098 [arXiv: 1803.00060] [INSPIRE].

[31] A.N. Khan, W. Rodejohann and X.-J. Xu, Borexino and general neutrino interactions, Phys. Rev. D 101 (2020) 055047 [arXiv: 1906.12102] [INSPIRE].

[32] K. Jodłowski and S. Trojanowski, Neutrino beam-dump experiment with FASER at the LHC, arXiv: 2011.04751 [INSPIRE].

[33] B. Batell, J.L. Feng and S. Trojanowski, Detecting dark matter with far-forward emulsion and liquid argon detectors at the LHC, Phys. Rev. D 103 (2021) 075023 [arXiv:2101.10338] [INSPIRE].

[34] R.C. Allen et al., Study of electron-neutrino electron elastic scattering at LAMPF, Phys. Rev. D 47 (1993) 11 [INSPIRE].

[35] LSND collaboration, Measurement of electron-neutrino-electron elastic scattering, Phys. Rev. D 63 (2001) 112001 [hep-ex/0101039] [INSPIRE]. 
[36] MINERvA collaboration, Measurement of neutrino flux from neutrino-electron elastic scattering, Phys. Rev. D 93 (2016) 112007 [arXiv: 1512.07699] [INSPIRE].

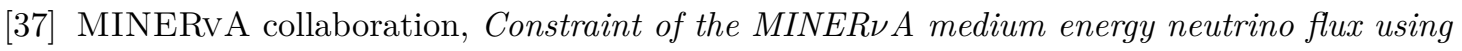
neutrino-electron elastic scattering, Phys. Rev. D 100 (2019) 092001 [arXiv:1906.00111] [INSPIRE].

[38] W. Rodejohann, X.-J. Xu and C.E. Yaguna, Distinguishing between Dirac and Majorana neutrinos in the presence of general interactions, JHEP 05 (2017) 024 [arXiv:1702.05721] [INSPIRE].

[39] C.A. Argüelles, M. Hostert and Y.-D. Tsai, Testing new physics explanations of the MiniBooNE anomaly at neutrino scattering experiments, Phys. Rev. Lett. 123 (2019) 261801 [arXiv: 1812.08768] [INSPIRE].

[40] J. Liao, D. Marfatia and K. Whisnant, Nonstandard interactions in solar neutrino oscillations with Hyper-Kamiokande and JUNO, Phys. Lett. B 771 (2017) 247 [arXiv: 1704.04711] [INSPIRE].

[41] BoreXINo collaboration, Absence of day-night asymmetry of $862 \mathrm{keV}^{7}$ Be solar neutrino rate in Borexino and MSW oscillation parameters, Phys. Lett. B 707 (2012) 22 [arXiv: 1104.2150].

[42] G. Bellini et al., Precision measurement of the ${ }^{7}$ Be solar neutrino interaction rate in Borexino, Phys. Rev. Lett. 107 (2011) 141302 [arXiv:1104.1816] [InSPIRE].

[43] Borexino collaboration, First evidence of pep solar neutrinos by direct detection in Borexino, Phys. Rev. Lett. 108 (2012) 051302 [arXiv:1110.3230] [InSPIRE].

[44] Borexino collaboration, Final results of Borexino Phase-I on low energy solar neutrino spectroscopy, Phys. Rev. D 89 (2014) 112007 [arXiv: 1308.0443] [InSPIRE].

[45] BOREXINO collaboration, Neutrinos from the primary proton-proton fusion process in the Sun, Nature 512 (2014) 383 [INSPIRE].

[46] Borexino collaboration, Improved measurement of ${ }^{8} \mathrm{~B}$ solar neutrinos with $1.5 \mathrm{kt} \cdot \mathrm{y}$ of Borexino exposure, Phys. Rev. D 101 (2020) 062001 [arXiv:1709.00756] [INSPIRE].

[47] N. Vinyoles et al., A new generation of standard solar models, Astrophys. J. 835 (2017) 202 [arXiv: 1611.09867] [INSPIRE].

[48] C. Boehm, M.J. Dolan and C. McCabe, A lower bound on the mass of cold thermal dark matter from Planck, JCAP 08 (2013) 041 [arXiv: 1303.6270] [INSPIRE].

[49] K. Kannike, M. Raidal, H. Veermäe, A. Strumia and D. Teresi, Dark matter and the XENON1T electron recoil excess, Phys. Rev. D 102 (2020) 095002 [arXiv:2006.10735] [INSPIRE].

[50] R. Essig, J. Mardon and T. Volansky, Direct detection of sub-GeV dark matter, Phys. Rev. D 85 (2012) 076007 [arXiv: 1108.5383] [INSPIRE].

[51] R. Essig, M. Fernandez-Serra, J. Mardon, A. Soto, T. Volansky and T.-T. Yu, Direct detection of sub-GeV dark matter with semiconductor targets, JHEP 05 (2016) 046 [arXiv: 1509.01598] [INSPIRE].

[52] LZ collaboration, The LUX-ZEPLIN (LZ) experiment, Nucl. Instrum. Meth. A 953 (2020) 163047 [arXiv: 1910.09124].

[53] Planck collaboration, Planck 2015 results. XIII. Cosmological parameters, Astron. Astrophys. 594 (2016) A13 [arXiv:1502.01589] [INSPIRE]. 
[54] H. Yuksel, J.F. Beacom and C.R. Watson, Strong upper limits on sterile neutrino warm dark matter, Phys. Rev. Lett. 101 (2008) 121301 [arXiv:0706.4084] [InSPIRE].

[55] A. Boyarsky, D. Malyshev, A. Neronov and O. Ruchayskiy, Constraining DM properties with SPI, Mon. Not. Roy. Astron. Soc. 387 (2008) 1345 [arXiv:0710.4922].

[56] R. Essig, E. Kuflik, S.D. McDermott, T. Volansky and K.M. Zurek, Constraining light dark matter with diffuse X-ray and gamma-ray observations, JHEP 11 (2013) 193 [arXiv: 1309.4091] [INSPIRE].

[57] K. Perez, K.C.Y. Ng, J.F. Beacom, C. Hersh, S. Horiuchi and R. Krivonos, Almost closing the $\nu$ MSM sterile neutrino dark matter window with NuSTAR, Phys. Rev. D 95 (2017) 123002 [arXiv: 1609.00667] [INSPIRE].

[58] B.M. Roach et al., NuSTAR tests of sterile-neutrino dark matter: new galactic bulge observations and combined impact, Phys. Rev. D 101 (2020) 103011 [arXiv:1908.09037] [INSPIRE]. 